\title{
DETECTION ET CARACTERISATION DES BACTERIES PHYTOPATHOGENES TRANSMISES PAR LES SEMENCES DU RIZ AU BURKINA FASO
}

\author{
S.L. OUEDRAOGO ${ }^{1}$, I. SOMDA ${ }^{2}$, F. BORRO ${ }^{3}$ et Y. SERE ${ }^{4}$ \\ ${ }^{1}$ Institut de l'Environnement et de Recherches Agricoles (INERA) Station de Di, \\ BP 910 Bobo Dioulasso Burkina Faso. e-mail : alsanou@fasonet.bf \\ 2 Institut du Développement Rural (IDR). 01 BP 1091 Bobo -Dioulasso 01 Burkina Faso. \\ ${ }^{3}$ Centre Agricole Polyvalent de Matourkou (CAP/M) Bobo- Dioulasso, Burkina Faso. \\ ${ }^{4}$ Association pour le Développement de la Riziculture en Afrique de l'Ouest (ADRAO/WARDA) \\ 01 BP 2551 Bouaké 01 Côte d'Ivoire.
}

\begin{abstract}
RESUME
Douze échantillons de riz provenant de différentes régions du Burkina Faso ont été testés en vue de faire un inventaire des bactéries phytopathogènes transmises par les semences du riz. La méthode de détection en milieu liquide (Liquid assay) utilisée à I'Institut Danois de Pathologie de Semences a été adoptée pour l'isolement des bactéries. Des observations sur la fluorescence, la coloration et la forme des colonies en comparaison avec les bactéries de références ont été faites pour le choix des colonies suspectes. Le profil biochimique des colonies choisies a été utilisé pour l'identification des bactéries au niveau de l'espèce. Cette identité a été confirmée par les tests de pathogénie sur les plantules de riz. $92 \%$ des échantillons de semences testées ont été infestées par les bactéries. Acidovorax avenae subsp. avenae ainsi que Xanthomonas oryzae pv. oryzae ont été chacune détectées dans $50 \%$ des échantillons et Burkholderia Glumae dans 16,66 \%. $52 \%$ des bactéries identifiées se rapportent à Acidovorax avenae subsp. avenae, $35 \%$ à Xanthomonas oryzae pv. oryzae et $13 \%$ à Burkholderia glumae. Pseudomonas fuscovaginae et Pseudomonas syringae pv syringae n'ont pas été rencontrés dans les échantillons étudiés.
\end{abstract}

Mots clés : Bactéries, détection, semences, riz, Burkina Faso.

\section{ABSTRACT}

Twelve seed samples of rice from various localities of Burkina Faso were screened for the presence of seed-transmitted plant pathogenic bacteria. The liquid assay used at the Danish Government Institute of Seed Pathology for Developping Countries was the method used for the extraction of bacteria. The observation of the fluorescence on King's B medium, the colour and colonies morphology in comparison with the reference were the methods used for the choice of suspected bacteria. Identification of colonies was performed on biochemical characteristic and pathogenicity tests. $92 \%$ percent of seed samples tested were infected by bacteria. Acidovorax avenae subsp. avenae as well as Xanthomonas oryzae pv. oryzae were each detected in $50 \%$ of seed samples and Burkholderia glumae in $16.66 \%$. $52 \%$ of bacteria belong to Acidovorax avenae subsp. avenae, $35 \%$ to Xanthomonas oryzae $p v$. oryzae and $13 \%$ to Burkholderia glumae. Pseudomonas fuscovaginae and Pseudomonas syringae pv syringae were not detected in the seed samples tested.

Keywords : Bacteria, detection, seeds, rice, Burkina Faso. 


\section{INTRODUCTION}

Le riz (Oryza sativa L.) est l'une des principales céréales cultivées dans le monde (Agarwal, 1994). Selon les données de Anonyme 2 (1998), la production mondiale du riz paddy s'élève à 563188000 tonnes avec un rendement moyen de 3,74 t/ha. La Thaïlande et les Etats-Unis sont les principaux exportateurs tandis que l'Afrique au Sud du Sahara et le Moyen-Orient demeurent les principaux importateurs.

La production du riz est entravée par de nombreux aléas biotiques parmi lesquels dominent les maladies, les insectes, les nématodes et les adventices (Brenière, 1983; Dembele, 2001). Sy et Sere, (1996) indiquent que les maladies sont considérées comme les plus importantes causes de la diminution de la quantité et de la qualité des produits.

Au Burkina Faso, d'importants travaux de recherche ont été réalisés dans des disciplines telles que la mycologie, la virologie et l'entomologie. Par contre, beaucoup d'efforts restent à faire en bactériologie, eu égard aux pertes considérables occasionnées sur le riz à Bagré en 1998, où $50 \%$ de dégâts causés par le fléchissement bactérien ont été enregistrés sur une variété sensible introduite dans le périmètre.
De plus on dispose de très peu d'informations dans le pays sur les bactérioses transmises par les semences du riz, notamment Xanthomonas oryzae pv oryzae (Srivastava et Rao, 1964), Xanthomonas oryzae pv. oryzicola (Shekhawat et al., 1969 ; Kihupi, 1997), Acidovorax avenae subsp. avenae (Shakya et al.,1986 ; Kihupi et al.,1999), Burkholderia glumae (Goto et al.,1987), Pseudomonas fuscovaginae (Zeigler et Alvarez, 1986 et 1987).

Le but de cette étude, est d'évaluer la prévalance des différentes bactéries ci-dessus mentionnées dans les semences du riz cultivé au Burkina Faso et proposer des moyens de contrôle des dégâts.

\section{MATERIEL ET METHODES}

\section{MATERIEL}

Le matériel biologique utilisé est constitué de la semence de riz, des plants de tabac et des bactéries de référence.

\section{Semences de riz}

Le tableau 1 fournit les informations sur les semences collectées.

Tableau 1 : Variétés, lieux, et année de collecte des semences de riz.

Varieties, localities and year of collection of rice seeds.

\begin{tabular}{cccccc}
\hline$N^{\circ}$ de l'échantillon & $\begin{array}{c}\text { Lieux de } \\
\text { collecte }\end{array}$ & $\begin{array}{c}\text { Année de } \\
\text { collecte }\end{array}$ & Variétés & $\begin{array}{c}\text { Poids des } \\
\text { échantillons } \\
\text { en Kg }\end{array}$ & $\begin{array}{c}\text { Nombre de } \\
\text { grains } \\
\text { testés }\end{array}$ \\
\hline 01 & Karfiguéla & SH 2000 & Locale & 1,409 & 400 \\
02 & Karfiguéla & SH 2000 & Locale & 1,540 & 400 \\
03 & Vallée du Kou & SH 2000 & Gambiaka & 1,637 & 400 \\
04 & Vallée du Kou & SH 2000 & Locale & 1,598 & 400 \\
05 & Banzon & SH 2000 & SC 27 & 2,550 & 400 \\
06 & Banzon & SH 2000 & Locale & 2,100 & 400 \\
07 & Bagré & SH 2000 & FKR19 & 2,650 & 400 \\
08 & Bagré & SH 2000 & FKR19 & 1,424 & 400 \\
09 & Sourou (AMVS) & SH 2000 & I I TA123 & 1,405 & 400 \\
10 & Sourou (AMVS) & SH 2000 & I I TA123 & 1,365 & 400 \\
11 & Ouahigouya & SH 2000 & Locale & 1,040 & 400 \\
12 & Ouahigouya & SH 2000 & Locale & 0,945 & 400 \\
\hline
\end{tabular}

S.H. 2000 = Saison humide $2000 ;$ AMVS = Autorité de Mise en Valeur du Sourou. 


\section{Plants de tabac}

La variété $P$ x Clarge utilisée provient de Mangodara (Burkina Faso) et de la récolte de 1998. Elle nous a été gracieusement offerte par la Société MABUCIG de Bobo-Dioulasso.

\section{Bactéries de référence}

Les différentes bactéries de références utilisées au cours des manipulations sont :

Pseudomonas syringae pv syringae, NCPPB 1770 / 22071997 ;

Acidovorax avena subsp avenae, NEPAL;

Pseudomonas glumae, NCPPB, 2391, Juillet 1998 ;

Xanthomonas oryzae pv oryzae NCPPB 1150 ;

Xanthomonas oryzae pv oryzicola NCPPB 1585.

Ces bactéries de références ont été gracieusement fournies par l'Institut Danois de Pathologie des Semences (Copenhague).

\section{Milieux de culture}

Plusieurs milieux de culture ont été utilisés. Ce sont : Le Bouillon nutritif (BN), l'Agar nutritif (NA), l'Agar nutritif + Sucrose (NAS), King's B (KB), Facteur de Croissance (GF), Oxydation et Fermentation (O/F), Yeast Dextrose Calcium carbonate agar (YDC), Amidon (SM) et Lugole (LM).

\section{METHODES}

\section{Collecte et conservation des semences}

Plusieurs variétés locales et améliorées de riz, non traitées provenant de six zones différentes de production rizicole (Sourou, Bagré, Ouahigouya, Banzon, Vallée du Kou, Karfiguela) ont été collectées. La collecte a été faite directement chez les producteurs. Le poids des échantillons a été compris entre 1 et $2,5 \mathrm{~kg}$ (tableau 1). Tous les échantillons on été colloctés pendant la saison humide 2000. Les différents échantillons ont été regroupés par zone de production et conservés dans une chambre froide à I'INERA/Di afin préserver les germes pathogènes des semences.

\section{Extraction des bactéries}

Quatre cents grains de riz ont été pesés et broyés dans un broyeur-mixeur (Commercial Blender). La farine a été collectée dans une bouteille contenant $200 \mathrm{ml}$ d'eau distillée à laquelle $1,70 \mathrm{~g}$ de $\mathrm{NaCl}$ ont été ajoutés pour obtenir une solution saline à $0,85 \% \mathrm{de} \mathrm{NaCl}$. La suspension obtenue a été incubée pendant deux heures à la température ambiante au laboratoire et la bouteille agitée toutes les $30 \mathrm{mn}$. A l'aide d'une pipette graduée, $9 \mathrm{ml}$ de bouillon nutritif ont été mesurés et introduits dans des flacons puis stérilisés. 4 séries de dilutions à concentrations décroissantes ( $4 x: 10)$ ont été ensuite effectuées à partir de ces flacons. En effet, $1 \mathrm{ml}$ de solution aqueuse a été prélevée dans la suspension de départ et ajouté au contenu du premier flacon. Ce flacon a constitué alors la première dilution $\left(10^{-1}\right)$. Un millilitre de ce dernier a été également utilisé pour préparer la deuxième dilution et la même procédure appliquée jusqu'à l'obtention de la quatrième dilution. $50 \mu \mathrm{l}$ de chaque dilution, y compris la suspension non diluée, sont prélevés à l'aide d'une pipette et introduits dans les boîtes de Pétri. Les boîtes sont renversées et mises en incubation pendant 3 à 5 jours à $28-30^{\circ} \mathrm{C}$, selon les bactéries recherchées. A l'issue cette période commence le choix des colonies suspectes et ensuite l'identification.

\section{Identification des isolats bactériens}

Les souches ont été identifiées selon leur morphologie sur le milieu NA, la production des pigments fluorescents sur le milieu KB (King et al., 1954), la réaction de GRAM basée sur la solubilité dans l'hydroxyde de potassium à $3 \%$ (Lelliot and Stead, 1987), le test de pathogénicité sur le riz variété TCS10 (Shakya and Chung, 1983), la réaction d'oxydase (Kovacs,1956 ; Hildebrand and Schroth, 1968 ; Schaad, 1988), la production de levane, l'hydrolyse de la gélatine (Fahy and Persley, 1983 ; Schaad, 1988), I'hypersensibilité sur le tabac (Klement, 1983) et le test d'Oxydation/Fermentation (Hugh and Leifson, 1953). 


\section{Nombre de colonies bactériennes formées par unité de volume}

Le CFU est le nombre de colonies bactériennes formées par unités de volume (ml).

Quand la méthode de culture sur milieu agar (étalement à l'aide de spatule) est utilisée, le nombre de colonies pathogènes et de colonies saprophytes doit être déterminé. Selon I'Organisation Internationale pour la Standardisation (Anonyme 1, 1985) les boîtes de Pétri devraient être éliminées s'il n'y a pas de colonies bien séparées, au moins dans la moitié de la boîte. Pour que le résultat soit valable, en général, on exige que chaque boîte contienne au moins 15 à 150 colonies.

Le calcul du nombre de micro-organismes présents dans un échantillon par unité de volume $(C F U / \mathrm{ml})$ est obtenu à l'aide de la moyenne pondérée, du nombre de colonies

\section{RÉSULTATS ET DISCUSSION}

Douze échantillons de riz de plusieurs zones de production du Burkina Faso ont été évalués pour la présence des bactéries phytopathogènes transmises par les semences. L'identification des souches a été faite à l'aide des caractéristiques morphologiques et biochimiques, (tableau 2) ainsi que des tests de pathogénécité sur des plantules de riz. Trois espèces de bactéries ont été identifiées. Il s'agit de Xanthomonas oryzae pv. oryzae, Acidovorax avenae subsp. avenae et de Burkholderia glumae. Elles ont été rencontrées dans toutes les zones de culture (tableau 3). $92 \%$ des échantillons testés ont été infectés par un ou plusieurs agents pathogènes. Ces données font ressortir l'importance des bactérioses du riz au Burkina Faso. La poursuite des travaux de détection pour couvrir toutes les zones rizicoles du pays devient impérative. Outre le fait que l'infection existe dans toutes les zones prospectées, il est intéressant de noter que le niveau de l'infestation est très élevé (tableau 4). Le nombre de colonies bactériennes formées par $\mathrm{ml}$ (CFU/ml) est très élevé quelle que soit la catégorie de colonie considérée (colonies totales ou colonies suspectes). Cela montre qu'en matière de protection des plantes beaucoup reste à faire dans le domaine de la bactériologie. Au Burkina Faso, le niveau de deux dilutions successives en utilisant la formule suivante empruntée de l'ISO 7213.

$$
\mathrm{CFU}=\frac{\sum \mathrm{C}}{\mathrm{V}(\mathrm{n} 1+0,1 \mathrm{n} 2) \mathrm{d}}
$$

$\sum \mathrm{C}=$ La somme des colonies dans toutes les boîtes de Pétri dans lesquelles des organismes ont été identifiés.

$d$ = Dilution à partir de laquelle le premier comptage a pu être obtenu.

n1 = Nombre de boîtes de Pétri de la première dilution dans lesquelles les colonies ont été comptées.

n2 = Nombre de boîtes de Pétri de la $2^{\mathrm{e}}$ dilution dans lesquelles les colonies ont été comptées.

$\mathrm{V}=$ Volume (en $\mathrm{ml}$ ) de l'inoculum appliqué à chaque boîte de Pétri. Dans notre cas, $\mathrm{V}=0,050 \mathrm{ml}$.

d'infestation des semences par les bactéries est effectivement important quel que soit le type de semence. Des semences de tomate testées pour la présence de Ralstonia solanacearum avaient des CFU/ml de l'ordre de $10^{4}-10^{5}$ (Ouedraogo, 1998). Ces résultats sont semblables à ceux obtenus sur le riz $\left(10^{4}-10^{6}\right)$. Par comparaison avec les tests effectués sur des semences en provenance de la Tanzanie, les semences du Burkina Faso sont beaucoup plus infestées. En effet Nancy (communication personnelle) a obtenu des CFU/ml de l'ordre de 10 à $10^{3}$ sur les semences de tomate.

Nos résultats montrent que Acidovorax avenae subsp. avenae, est la bactérie la plus rencontrée. $50 \%$ des échantillons testés ont été infectés par cette bactérie. $52 \%$ de toutes les colonies bactériennes suspectées ont été identifiées comme appartenant au genre Acidovorax. avenae subsp. avenae. Somida et al. (2001) a indiqué que sur 10 échantillons de semences de riz du Burkina testés, 9 ont été infectés par Acidovorax. avenae subsp. avenae et l'incidence de la maladie a varié entre 5 et $20 \%$. Cette forte présence de la bactérie montre qu'au Burkina Faso, les conditions pédo climatique seraient favorables au développement de l'agent pathogène. Ainsi, il est fort possible que la plupart des variétés de riz cultivées dans le pays présentent une certaine sensibilité à la bactérie. De plus, très peu de méthodes de 


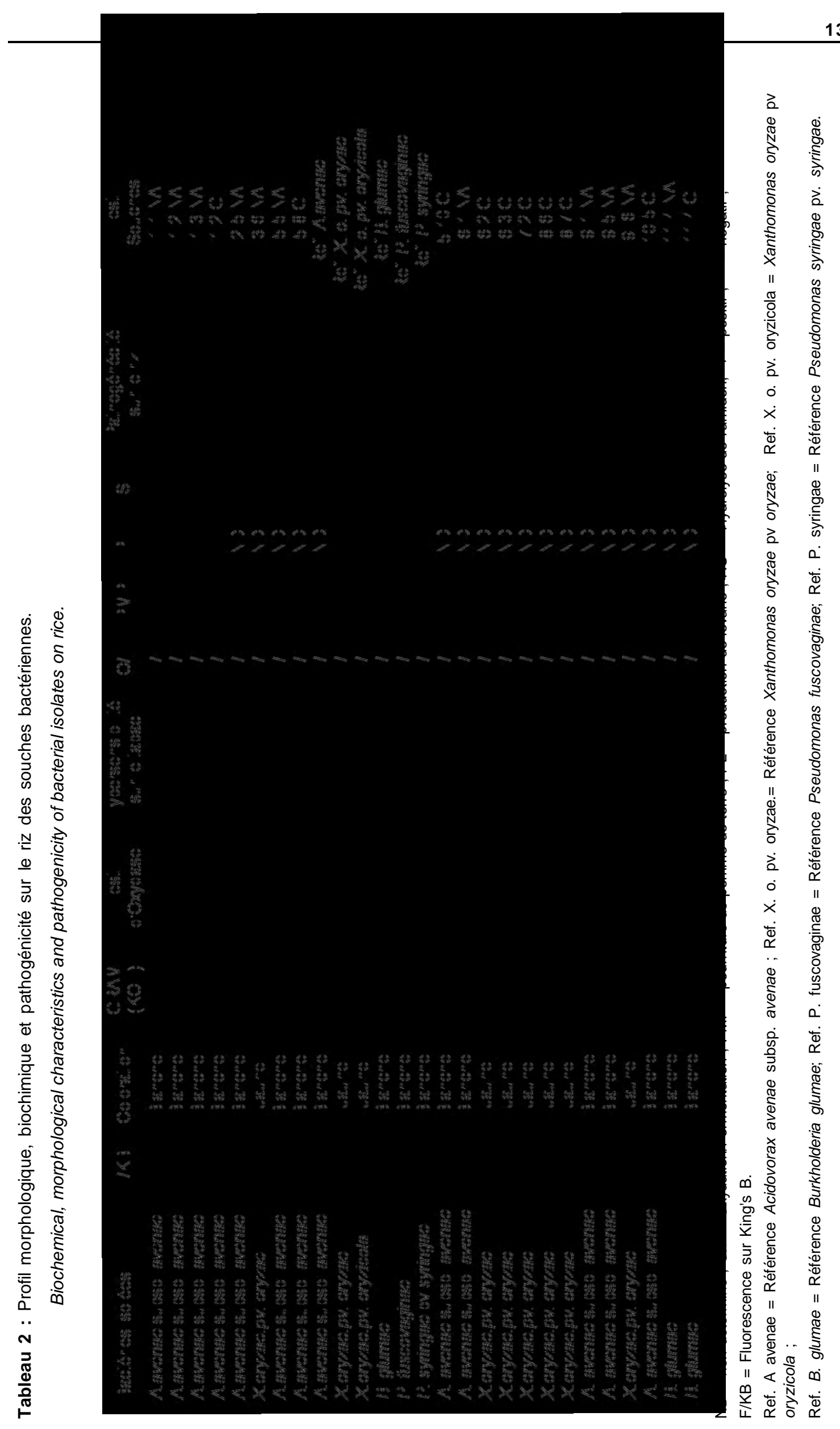

Agronomie Africaine 16 (2) : 9 - 17 (2004) 
Tableau 3 : Synthèse des bactéries détectées et leurs lieux de provenance.

Synthesis of detected bacteria and their origin.

\begin{tabular}{|c|c|c|c|}
\hline Bactéries isolées & Localités & Variétés & Souches \\
\hline Acidovorax avenae subsp. avenae & Karfiguela & Locale & $1-1 \mathrm{NA}$ \\
\hline Acidovorax avenae subsp. avenae & Karfiguela & Locale & $1-2 \mathrm{NA}$ \\
\hline Acidovorax avenae subsp. avenae & Karfiguela & Locale & $1-3 \mathrm{NA}$ \\
\hline Acidovorax avenae subsp. avenae & Karfiguela & Locale & $1-2 \mathrm{GF}$ \\
\hline Acidovorax avenae subsp. avenae & Karfiguela & Locale & $2-5 \mathrm{NA}$ \\
\hline Xanthomonas oryzae pv. oryzae & Vallée du Kou & Gambiaka FKR2 & 3-6 NA \\
\hline Xanthomonas oryzae pv. oryzae & Banzon & SC 27 & 5-5 NA \\
\hline Acidovorax avenae subsp. avenae & Banzon & SC 27 & 5-8 GF \\
\hline Acidovorax avenae subsp. avenae & Banzon & SC 27 & 5-10 GF \\
\hline Acidovorax avenae subsp. avenae & Banzon & Locale & 6-1 NA \\
\hline Xanthomonas oryzae pv. oryzae & Banzon & Locale & 6-2 GF \\
\hline Xanthomonas oryzae pv. oryzae & Banzon & Locale & 6-3 GF \\
\hline Xanthomonas oryzae pv. oryzae & Bagré & FKR 19 & $7-2 \mathrm{GF}$ \\
\hline Xanthomonas oryzae pv. oryzae & Bagré & FKR 19 & $8-6$ GF \\
\hline Xanthomonas oryzae pv.oryzae & Bagré & FKR 19 & 8-7 GF \\
\hline Acidovorax avenae subsp. avenae & Sourou (AMVS) & ITA 123 & $9-1 \mathrm{NA}$ \\
\hline Acidovorax avenae subsp. avenae & Sourou (AMVS) & ITA 123 & 9-5 NA \\
\hline Xanthomonas oryzae pv. oryzae & Sourou (AMVS) & ITA 123 & $9-6 \mathrm{NA}$ \\
\hline Acidovorax avenae subsp. avenae & Sourou (AMVS) & ITA 123 & $10-5 \mathrm{GF}$ \\
\hline Burkholderia glumae & Ouahigouya & Locale & 11-4 NA \\
\hline Burkholderia glumae & Ouahigouya & Locale & $11-4 \mathrm{GF}$ \\
\hline Burkholderia Glumae & Ouahigouya & Locale & $12-2 \mathrm{NA}$ \\
\hline Xanthomonas oryzae pv. oryzae & Ouahigouya & Locale & 12-6 NA \\
\hline
\end{tabular}

Tableau 4 : Récapitulatif du nombre de colonies bactériennes (cfu /ml) dans différents milieux de culture. Number of colonies (cfu/ml) in different culture media.

\begin{tabular}{|c|c|c|c|c|}
\hline Milieux & $\begin{array}{l}\text { Nombre total de } \\
\text { colonies }\end{array}$ & $\begin{array}{c}\text { Nombre de } \\
\text { colonies suspectes }\end{array}$ & $\begin{array}{l}\text { Total colonies } \\
(\mathrm{Cfu} / \mathrm{ml} \\
\left.\times 10^{5}\right)\end{array}$ & $\begin{array}{c}\text { Colonies suspectes } \\
(\mathrm{Cfu} / \mathrm{ml} \\
\left.\times 10^{2}\right) \\
\end{array}$ \\
\hline \multicolumn{5}{|l|}{ Echantillon I } \\
\hline NA & 209 & 03 & 20 & ND \\
\hline GF & 207 & 06 & 3,1 & ND \\
\hline YDC & 256 & 06 & ND & ND \\
\hline \multicolumn{5}{|l|}{ Echantillon 2} \\
\hline NA & 173 & 10 & 10 & 15 \\
\hline GF & 369 & 05 & 22 & 83 \\
\hline \multicolumn{5}{|l|}{ Echantillon 3} \\
\hline NA & 512 & 05 & 3,2 & 5,0 \\
\hline GF & 153 & 04 & 10 & ND \\
\hline \multicolumn{5}{|l|}{ Echantillon 4} \\
\hline NA & 241 & 05 & 20 & 43 \\
\hline GF & 868 & 04 & 52 & 70 \\
\hline \multicolumn{5}{|l|}{ Echantillon 5} \\
\hline NA & 307 & 07 & 0,47 & 6,6 \\
\hline GF & 281 & 09 & 3,0 & 1,6 \\
\hline \multicolumn{5}{|l|}{ Echantillon 6} \\
\hline NA & 149 & 09 & 9,0 & 16 \\
\hline GF & 178 & 06 & 10 & ND \\
\hline \multicolumn{5}{|l|}{ Echantillon 7} \\
\hline NA & 446 & 09 & 3,0 & 8,6 \\
\hline GF & 253 & & 4,0 & 70 \\
\hline \multicolumn{5}{|l|}{ Echantillon 8} \\
\hline NA & 209 & 10 & 3,4 & 64 \\
\hline GF & 66 & 10 & 8,0 & 150 \\
\hline \multicolumn{5}{|l|}{ Echantillon 9} \\
\hline NA & ND & 08 & ND & 12 \\
\hline GF & ND & 08 & ND & 72 \\
\hline \multicolumn{5}{|l|}{ Echantillon 10} \\
\hline NA & 301 & 12 & 18 & 7,7 \\
\hline GF & 316 & 06 & 30 & 340 \\
\hline \multicolumn{5}{|l|}{ Echantillon 11} \\
\hline NA & ND & 12 & ND & 75 \\
\hline GF & ND & 06 & ND & 100 \\
\hline \multicolumn{5}{|l|}{ Echantillon 12} \\
\hline NA & 314 & 12 & 54 & 140 \\
\hline GF & 235 & 04 & 14 & 66 \\
\hline
\end{tabular}

ND : non déterminé. 
contrôle de la bactériose ont été utilisées dans le pays. Cela expliquerait sa forte expansion.

Nos résultats sont en accord avec ceux obtenus par Kihupi (1997) en Tanzanie. L'auteur a indiqué, que $63 \%$ des échantillons testés d'origine Tanzanienne sont infectés par la bactérie. Des résultats similaires ont été obtenus par Shakya et al. (1985). Xanthomonas oryzae pv. oryzae a été présent dans $50 \%$ des échantillons de riz testés. Cette présence très forte montre l'importance économique de cette bactérie au Burkina Faso. Des résultats également semblables ont été signalés par Srivastava et Rao (1964) en Inde où 50 à $100 \%$ des semences de riz sont infectées par Xanthomonas oryzae pv. oryzae. 116 et 129 échantillons de riz provenant respectivement des régions tropicales et subtropicales de la Chine ont été testés par Xie et ses collaborateurs de 1993 à 1998. (Xie et al., 1999). Ces auteurs ont noté que la présence de Xanthomonas oryzae pv. oryzae et Xanthomonas oryzae pv. oryzicola est encore très forte dans les régions tropicales et subtropicales de la Chine. Xanthomonas oryzae pv. oryzae n'a pas été détecté à Karfiguela. Cela ne signifie pas qu'elle est absente dans cette région. Son absence dans les échantillons pourrait s'expliquer entre autre par un nombre très réduit. Selon Sié (Communication Personnelle) Xanthomonas oryzae pv. oryzae est présente à Karfiguela comme dans toutes les autres zones de culture du riz au Burkina Faso. La bactérie a été détectée dans tous les échantillons testés en provenance de Bagré. Cette présence remarquée serait certainement liée à l'explosion du feu bactérien causé par Xanthomonas oryzae pv. oryzae en 1998 à la suite de l'introduction d'une variété sensible du riz, la TCS 10 en provenance de la Chine. Ainsi, les autres variétés cultivées sur le périmètre seraient certainement contaminées d'où le niveau d'infestation élevé des échantillons collectés dans ce périmètre. La bactérie peut être contrôlée en traitant les semences au Brestanol 45 (chlorure de triphényl-étain, poudre mouillable) à la dose de $0,25 \mathrm{~g} / 100 \mathrm{~g}$ de semences (Singh et Rao, 1982). Burkholderia glumae est détecté sur 16,66 \% des échantillons testés. C'est la première fois que cette bactérie a été détectée dans les semences du riz au Burkina Faso. Les caractéristiques morphologiques et biochimiques sont semblables à celles de la bactérie de référence. Le pathogène a été observé dans la partie nord du Burkina Faso et les investigations doivent se poursuivre pour confirmer sa présence. Burkholderia glumae peut être contrôlée en trempant les graines de riz dans l'eau chaude à $65^{\circ} \mathrm{C}$ pendant 6 jours (Ziegler et Alvarez, 1988). Il en est de même pour Acidovorax avenae subsp. avenae.

Deux bactéries transmises par les semences du riz n'ont pas été détectées. Il s'agit de Pseudomonas syringae pv. syringae et de Pseudomonas fuscovaginae. L'absence de ces deux bactéries dans les semences s'expliquerait par le fait que : 1) les deux bactéries ne seraient pas dans les échantillons de semences testées ; 2) il est possible que les milieux de cultures ne soient pas suffisamment sélectifs pour leur détection ; 3) le nombre d'échantillons testés est réduit.

\section{CONCLUSION}

Cette étude a permis de montrer l'importance des bactérioses transmises par les semences du riz à travers le taux d'infection des échantillons testés et leur zone de provenance. Toutes les zones de culture dans lesquelles les semences ont été prélevées sont infectées. Il est indispensable que des précautions adéquates soient prises pour améliorer l'état sanitaire des semences avant le semis. Les recherches futures doivent être orientées sur l'établissement du niveau de tolérance des bactéries détectées et des méthodes simples de désinfection des semences pour un contrôle phytosanitaire plus efficace du riz au Burkina Faso. 


\section{REFERENCES}

AGARWAL (P. C.), (N. C) MORTENSEN, (S. B.) MATHUR. 1994. Maladies du riz transmises par les semences et tests phytosanitaires. CTA - ADRAO, $95 \mathrm{p}$.

ANONYME1. 1998. Annuaire statistique, FAO. Volume 52. Rome. 64-65.

ANONYME 2. 1996. International Standard 7218. Microbiology-General guidance for microbiological examinations ISO ( $2^{\text {nd }}$ edition). Ref. No. ISO 7218-1996.UDC 579.678. International Organization of Standardization, Geneva, Switzerland, $43 \mathrm{p}$.

BRENIERE (J.). 1983. Principaux ennemis du riz en Afrique de l'ouest et leur contrôle. $2^{\text {nd }}$ édition. Monrovia. Libéria, ADRAO Monrovia, $87 \mathrm{p}$.

DEMBELE (K.). 2001. Caractérisation de variétés de riz vis-à-vis de la pyriculariose à l'Ouest et au Sud-Ouest du Burkina Faso. Mémoire de fin d'étude, IDR Bobo-Dioulasso Burkina Faso, 1-9.

FAHY (P. C.) and (G. J.) PERSLEY. 1983. Plants bacterial diseases : A diagnostic guide. Academic Press, London, $393 \mathrm{p}$.

GOTO (M.), (K.) NISHIYAMA and (K.) OHATA. 1987. Bacteria causing grain rot of rice. Annals Phytopathol. Soc. Japan 53, 141-149.

HILDEBRAND (D. C.) and (M. N.) SCHROTH. 1968. Removal of pseudomonas from plants leaves and measurement of their in vivoglucosidase synthesis. Phytopathology 58 : 354-358.

HUGH (R.) and (E.) LEIFSON. 1953. The taxonomic significance of fermentative versus oxidative metabolism of carbohydrates by various Gram-negative bacteria. Journal of bacteriology $66: 24-6$.

KIHUPI (A. L.). 1997. Detection and characterization of seed-borne pathogenic bacteria of rice cultivars from Tanzania. Training. report. Department of Crop Science and Production. Sokoine University of Agriculture, PO Box 3005, Morogoro. Tanzania, $64 \mathrm{p}$.

KIHUPI (A. L.), (R. B) MABAGALA and (C. N.) MORTENSEN. 1999. Occurence of Acidovorax avenae subsp. avenae in rice seeds in Tanzania. African Plant Protection 5(1) : 55-58.

KING (E. O.), (M.K) WARD and (D. E.) RANEY. 1954. Two simple media for the demonstration of pyocyanin and fluorescin. Journal of Laboratory Clinical Medecine $44: 301-307$
KLEMENT (Z.). 1983. Detection of seed-borne bacteria by hypersensititve reaction. Seed Science and Technology 11 : 589-593.

KOVAC'S (N.). 1956. Identification de Pseudomonas pyacyanea par la réaction d'oxydase. Nature. London. 178, 703 p.

LELLIOT (R. A.) and (D. E.) STEAD. 1987. Methods for the diagnosis of bacterial diseases of plants. Blackwell Scientific Publications, William Clowes, London : $216 \mathrm{p}$.

OUEDRAOGO (L.). 1998. Detection of phytopathogenic bacteria in seeds and stems of tomato, eggplant and pepper from Burkina Faso and Indonesia. Training report, Danish Government Institute of Seed Pathology for Developing Countries, (DGISP) Ryvangs Alle 78, Denmark, 41 p.

SCHAAD (N. W.). 1988. Laboratory guide for identification of plant pathogenic bacteria, $2^{\text {nd }}$ edition. APS, St Paul, $164 \mathrm{p}$.

SHAKYA (D. D.) and (H. S.) CHUNG. 1983. Detection of Pseudomonas avenae in rice seeds. Seed Science and Technology $11:$ 256-259.

SHAKYA (D. D.), (H. S.) CHUNG and (F.) VINTHER. 1986. Transmission of Pseudomonas avenae the cause of bacterial stripe of rice. Phytopathology $\mathrm{n}^{\circ} 116$ : 92-96

SHAKYA (D. D), (F.) VINTHER and (S. B.) MATHUR. 1985. World wide distribution of bacterial stripe pathogen of rice identified as Pseudomonas avenae. Phytopathologische zeitschrift. No $114: 256$ - 259.

SHEKHAWAT (G. S.), (D. N.) SRIVASTAVA and (Y.P.) RAO. 1969. Seed infection and transmission of bacterial leaf streak of rice. Plant Disease Reporter 53 : 115-116.

SINGH (R. S.) and (M. H. S.) RAO. 1982. Evaluation of chemical treatements for eradicating $X$. oryzae from rice seeds. Seed Science and Technology $10: 119-23$

SOMDA (I.), (M. S.) VEENA and (C. N.) MORTENSEN. 2001. First report on the occurrence of bacterial stripe organism Acidovorax avenae subsp. avenae in rice seeds from Burkina Faso. Plant Disease 85, $804 \mathrm{p}$.

SRIVASTAVA (D. N.) and (Y. P.) RAO. 1964. Seed transmission and epidemiology of the bacterial blight disease of rice in North India. Indian Phytopathology 17 : 77 - 78.

SY (A. A) et (Y.) SERE. 1996. Manuel de formation en pathologie du riz. ADRAO. Imprint design. Royaume Uni. 76 p.

XIE, GUALIN, ZHENG, JICHI and (T. V.) MEW. 1999. Pathological bacteria associated with rice seed in the subtropic and the tropic areas. Acta Agriculturae Zhejienensis, volume XI : 127 - 132. 
ZEIGLER (R. S.) and (E.) ALVAREZ. 1986. Bacterial sheath brown rot (BSBR) in Latin America. International Rice Research NEWS LETTER volume XI : 15 - 16.

ZEIGLER (R. S.) and (E.) ALVAREZ. 1987. Bacterial sheath brown rot caused by Pseudomonas fuscovaginae in Latin America. Plant Disease 71 : 592-597.

ZEIGLER (R. S.) and (E.) ALVAREZ. 1988. Pseudomonas spp. causing grain and sheath rot of rice in Latin America. Abstracts, $5^{\text {th }}$ Int. Congress of plant pathology. Kyoto, Japan, 20-27 August, 1988. Poster Section XIII 1-16, 411 p. 